\title{
Analyzing Control Traffic Overhead versus Mobility and Data Traffic Activity in Mobile Ad-hoc Network Protocols
}

\author{
Laurent Viennot, Philippe Jacquet and Thomas Heide Clausen* \\ INRIA Rocquencourt, Projet Hipercom, \\ Domaine de Voluceau, B.P.105, 78153 Le Chesnay cedex, France \\ Telephone: +33139635363 Fax: +33139635566 \\ Email: \{Laurent.Viennot, Philippe.Jacquet, Thomas.Clausen\}einria.fr
}

\begin{abstract}
This paper proposes a general, parameterized model for analyzing protocol control overhead in mobile ad-hoc networks. A probabilistic model for the network topology and the data traffic is proposed in order to estimate overhead due to control packets of routing protocols.

Our analytical model is validated by comparisons with simulations, both taken from literature and made specifically for this paper. For example, our model predicts linearity of control overhead with regard to mobility as observed in existing simulations results. We identify the model parameters for protocols like AODV, DSR and OLSR.

Our model then allows accurate predictions of which protocol will yield the lowest overhead depending on the node mobility and traffic activity pattern.
\end{abstract}

\section{INTRODUCTION}

Mobile ad-hoc networking (MANET) has experienced a growing interest since the apparition of affordable radio interfaces, allowing wireless connectivity of mobile nodes. A keypoint in connecting a group of mobile nodes is the design of a routing protocol that allows out-of-range nodes to communicate through the relaying of their traffic by intermediate nodes. This is the subject of the IETF MANET working group [5], [14] where several protocols are being proposed.

The different routing protocols can be divided into two disjoint classes, according to the way routes are created:

Reactive protocols find routes on demand when needed by a source. They usually rely on flooding when no topology information is available. I.e. the source floods a packet and the path followed by this packet to reach the destination is then used.

Proactive protocols proactively discover the topology with every node emitting regular hello packets and an optimized mechanism is used to broadcast local topology information.

These two approaches have different characteristics with regard to control traffic overhead. Reactive protocols generate overhead only when a new route is needed, while proactive protocols continuously generate control traffic. Link failure,

\footnotetext{
* Thomas Heide Clausen may also be contacted at MindPass Center for Distributed Systems, Department of Computer Science, Aalborg University, Fredrik Bajers Vej 7E 9220 Aalborg Ø, Denmark
}

mainly due to mobility, will produce additional overhead with both approaches since routes must be repaired as quickly as possible. In a reactive protocol, routes either have to be repaired or rediscovered. In a proactive protocol, the broadcasted topology in the network has to be updated to reflect the change.

Comparing the overhead from these two very different approaches is thus a challenging task. The objective of this paper is to propose a model to analyze control traffic overhead of MANET routing protocols in order to better identify which protocol is better suited for a particular situation. By control traffic overhead, we mean the bandwidth utilization due to control packets.

It is obvious, that control traffic overhead mainly depends (apart from the routing protocol used) on the topology (and its changes) and the data traffic. Our main result is a reasonably simple model for the relationship between control traffic overhead and both topology and traffic. We use simulations of real protocols to check that the predictions of the model correspond with the simulation results. This comparison is also needed to infer the protocol parameters of our model. (Each specific protocol optimization is modeled by two or three numbers which are more easily inferred by simulation.) Finally, this allows us to compare these protocols for all mobility and traffic activity patterns, even the cases not covered by simulations.

\begin{tabular}{|l|c|c|}
\cline { 2 - 3 } \multicolumn{1}{c|}{} & Reactive & Proactive \\
\hline Fixed & $\lambda o_{r} N^{2}$ & $h_{p} N+o_{p} t_{p} N^{2}$ \\
Mobility & $o_{r} \mu a L N^{2}$ & $o_{p} \mu A N_{p} N^{2}$ \\
\hline
\end{tabular}

TABLE I

GENERIC CONTROL TRAFFIC OVERHEAD OF BOTH PROTOCOL FLAVORS IN NUMBER OF PACKETS.

Our approach is analogous to complexity analysis: we focus on the main contribution term to control traffic overhead. Table I presents the estimation of control traffic overhead for the two main flavors of protocols. The main parameters are: $N$ the number of nodes, $\mu$ the failure rate of a link (which models mobility), and $a$ the number of active routes per node (which models activity of the traffic). 


\begin{tabular}{|l|l|}
\hline \multicolumn{2}{|c|}{ Network parameters } \\
\hline$N$ & number of nodes \\
$M$ & number of edges \\
$\Delta=2 M / N$ & average degree of a node \\
$\mu$ & link breakage rate (mobility) \\
$L$ & average length of a route \\
\hline
\end{tabular}

TABLE II

PARAMETERS DESCRIBING THE NETWORK.

\begin{tabular}{|c|c|}
\hline \multicolumn{2}{|r|}{ Traffic parameters } \\
\hline$\lambda$ & $\begin{array}{l}\text { route creation rate } \\
\text { per node }\end{array}$ \\
\hline$a$ & $\begin{array}{l}\text { number of active routes } \\
\text { per node (activity) }\end{array}$ \\
\hline
\end{tabular}

TABLE III

PARAMETERS DESCRIBING DATA TRAFFIC.
Reactive protocols include AODV [20] by C. Perkins et. al., TORA [19] by M. S. Corson and V. Park, DSR [13] by J.Broch, D.Johnson and D.Maltz, ODMRP [17] by S.-J. Lee, M. Gerla, and C.-C. Chiang, and RDMAR [1]. Most of these protocol optimize their flooding cost. These various optimizations are not analyzed in this paper.

Proactive protocols include OLSR [4] by Qayyum, Jacquet, Muhlethaler, Laouiti, Clausen and Viennot and TBRPF [2] by R. Ogier and B. Bellur. Finally, there are hybrid protocols such as ZRP [9] by Z.J. Haas and M.R. Pearlman, which try to take advantages of both the proactive and reactive approaches.

Applying our analytical model, we are going to see that proactive and reactive approaches may both overtake the other in terms of control overhead, depending on network and traffic profiles.

\section{A. Paper outline}

The organization of the remainder of this paper is as follows: a generic model is given in section II, taking into account network density, mobility, traffic creation and traffic density. Sections III and IV are devoted to estimating control traffic overhead by analyzing both the number of control packets and their bandwidth cost for generic versions of both reactive and proactive protocols. Section $\mathrm{V}$ compares our analysis to simulations of reactive protocols, taken from literature, as well as simulations of OLSR conducted for the purpose of this paper. Section VI discusses the analysis of protocol parameters of the model. Section VII compares OLSR to DSR with respect to mobility and traffic activity.

\section{MODEL FOR NETWORK, TRAFFIC AND PROTOCOLS}

To allow the analysis of different protocols, we propose a simple model. While the model is simple, we will see that existing simulations of routing protocols confirm the model. For simplicity we assume that no congestion occurs in the network (this assumption greatly simplifies the analysis of protocol behaviors since it implies that few control packets are lost).

\section{A. Network model parameters}

The parameters used to model the network are summarized by table II. $N$ denotes the number of nodes in the network, $M$ the number of edges. We consider that two nodes are linked by an edge if they are able to communicate directly, i.e. each one is then neighbor of the other. $\Delta$ is the average degree of a node, the degree being the number of neighbors of a node.
To model mobility, we introduce $\mu$, the average number of link breakage per link during a second. I.e. a link lasts on average $1 / \mu$ seconds. We assume that the link breakage is constant and that link creation balances link breakage. I.e. that $M$ is supposed to be constant. This implies that $M \mu$ links, in total, are created per second. Notice that it is logical to suppose that the total number of link creation or link breakage is proportional to the number of links.

Another parameter, depending mainly on the shape of the network, is the average length $L$ (number of hops) of a route.

We further make the assumption, that the above parameters remain constant, and that the network always remains connected.

\section{B. Traffic model parameters}

Concerning control traffic overhead, we mainly need to model data traffic creation and diversity. The parameters used to model the data traffic are summarized by table III. $\lambda$ denotes the average number of route creation by a node during a second. The average number of simultaneous active routes per node is denoted by $a$. An active route is a pair (source, destination) where the source continuously sends packets to the destination.

This is a rather simplistic traffic model, however we find that it is sufficient to compare the reactive and proactive approaches to ad hoc routing.

\section{Proactive protocol parameters}

A set of parameters depends on the protocol. We now provide an abstract description of the characteristics of proactive and reactive protocols, respectively. The descriptions are sufficiently detailed to allow reasoning about the protocols, and also sufficiently general to model any protocol, provided that the protocol parameters are correctly set.

Future work will be required to validate the model for each protocol and to identify the values of the parameters for each protocol by analysis rather than simulation. Notice that some parameters may depend on the topology of the network or the traffic pattern. However, the analysis gives satisfying results when compared to simulations found in the literature (see section $\mathrm{V}$ ).

Proactive protocols are relatively easy to model due the regularity of control packet emission.

Control packets mainly include packets for proactively discovering the local topology (usually called hello messages) and topology broadcast packets for allowing global knowledge of the topology. The parameters used to model the proactive protocols are summarized in table IV. $h_{p}$ and $t_{p}$ are respectively 


\begin{tabular}{|l|l|}
\hline \multicolumn{2}{|c|}{ Proactive protocols parameters } \\
\hline$h_{p}$ & hello rate \\
$H_{p}$ & average size of hello packets \\
$t_{p}$ & topology broadcast rate \\
$T_{p}$ & average size of topology broadcast packets \\
$o_{p}$ & broadcast optimization factor \\
$A N_{p}$ & active next hops ratio \\
\hline
\end{tabular}

TABLE IV

PROACTIVE PROTOCOL PARAMETERS

\begin{tabular}{|l|l|}
\hline \multicolumn{2}{|c|}{ Reactive protocols parameters } \\
\hline$h_{r}$ & hello rate (0 when possible) \\
$H_{r}$ & average size of hello packets \\
$R Q_{r}$ & average size of route request packets \\
$o_{r}$ & route request optimization factor \\
\hline
\end{tabular}

TABLE V

REACTIVE PROTOCOL PARAMETERS.

the number of hellos and broadcast information packets emitted by a node during a second. These parameters are expressed in terms of rates. $H_{p}$ denotes the average size of hello packets (typically $H_{p}=O(\Delta)$ ) and $T_{p}$ denotes the average size of the topology packets broadcasted by a node. We will see in section IV-B that a proactive protocol may need to send additional topology broadcast packets in order to react to topological changes. We introduce a parameter active next hop $A N_{p}$ to evaluate which topology changes may trigger additional control traffic. The active next hop is the average number of active links per node (when an active link breaks, a topology broadcast has to be carried out).

Proactive protocols can benefit from their knowledge of the topology in order to optimize broadcasting [15],[8],[2]. Ideally, $N / \Delta$ emissions are sufficient to broadcast a packet to every node, as compared to $N$ emissions for a complete flooding.

If $B_{p}$ denotes the average number of emissions to achieve a topology broadcast, we denote by $o_{p}$ the broadcast optimization factor, i.e. $o_{p}=B_{p} / N\left(1 / \Delta \leq o_{p} \leq 1\right)$. Estimating $T_{p}$ and $o_{p}$ are the main difficulty when describing a given proactive protocol.

\section{Reactive protocol parameters}

The parameters used to model the reactive protocols are summarized in table V. Reactive protocols may include hellos in order to detect link breakage. If hellos are used, $h_{r}$ denotes their rate and $H_{r}$ their size. Otherwise, information provided by the link layer is used to detect link breakage, in which case $h_{r}=0$ and $H_{r}=0$. The main contribution to control traffic overhead is due to the emission of route request and route reply messages. Route request packets are flooded by a source creating a route. Route reply packets are generally unicasted by the destination (or intermediate nodes that know a route to the destination) to the source, taking the path followed by the route request packet. To keep the model simple, we will not distinguish (regarding the cost of a route request) route reply packets from route request packets. This is acceptable since they usually have a comparable size and they are both triggered by route requests. $R Q_{r}$ will denote the average size of route request (and route reply) packets.

Some reactive protocols propose reduction of the flooding overhead by trying to limit the spread of flooding. This, e.g., by limiting the maximum number of retransmission (TTL) of the route request packet. This is often denoted expanding ring [7]. The danger of employing an expanding ring technique is that to reach a far destination, a node may have to initiate several floodings with increasing TTL. If $B_{r}$ is the average number of emissions for a route request (including route reply messages), we will denote by $o_{r}=B_{r} / N$ the route request optimization factor. With the expanded ring technique, beginning with a TTL 2 , we get $B_{r} \geq 1+\Delta$ and thus $d \leq o_{r} \leq k$ where $k$ is the maximum number of floodings for a route request. (Keep in mind that flooding costs at most $N$ emissions.) With pure flooding and a route reply from the destination, we get $o_{r}=1+L / N$ ( $L$ is the number of route reply messages in that case). When route caching is used, some route requests may be avoided, this should also be captured by $o_{r}$. The main difficulty in estimating the parameters of a given reactive protocol resides in $o_{r}$.

Alternatively, some protocols propose that the route reply be also flooded. This can also be captured with this parameter (with pure flooding, $o_{r}=2$ ).

Given these parameters, we are now able to analyze protocol overheads of both routing approaches.

\section{CONTROL TRAFFIC OVERHEAD IN FIXED NETWORK}

In this section, we will consider the control traffic overhead in a fixed network (i.e. supposing that there is no mobility). The additional cost of mobility is considered in the next section.

\section{A. Route creation overhead}

To create a route in a reactive protocol, the source initiates a route request. In our model, $\lambda N$ route requests are produced every second, producing $\lambda o_{r} N^{2}$ packets. This corresponds to a bandwidth overhead of $\lambda o_{r} R Q_{r} N^{2}$. Notice that using the same route from time to time may be considered as route creations since entries of a routing table have a timeout. If the period between two emissions on the same route is greater than this timeout, the second emission will produce a route request.

Indeed, since route requests are transmitted by flooding, any node in the network may receive a route to a source initiating a route request (not only the requested destination). That means that when a node needs a route for the first time to some destination, it may already know a route if the destination has recently initiated a route request. The route request then produces no control packet. This should be captured in the $o_{r}$ parameter. Notice that $o_{r}$ thus depends on the network and traffic parameters.

Proactive protocols have the advantage of having all routes ready for use and do not make any overhead at route creation. On the other hand, their fixed control traffic overhead includes the cost of route creation. 


\section{B. Fixed control traffic overhead}

With a proactive protocol, each node emits $h_{p}$ hello messages per second and initiate $t_{p}$ topology broadcast per second. This produces an overhead of $h_{p} N+t_{p} o_{p} N^{2}$ packets per second, corresponding to a bandwidth of $h_{p} H_{p} N+t_{p} o_{p} T_{p} N^{2}$.

If a reactive protocol uses hellos to detect link breakage, its hello overhead will be $h_{r} N$ packets per second, using a bandwidth of $h_{r} H_{r} N$. Notice that the size $H_{r}$ of reactive hello packets usually have constant size compared to a size proportional to $\Delta$ for proactive protocols where hello messages usually include the list of neighbors addresses.

\section{CONTROL TRAFFIC OVERHEAD DUE TO MOBILITY}

The most challenging task for our model is to quantify the emissions of control packets in reaction to mobility. Mobility is visible for the routing protocol through link creation and link breakage. MANET protocols do not usually generate additional control packets in reaction to link creation. However, it is very important to react quickly to link breakage when the link is actively being used for transferring data. A link breakage is detected either when some hellos are no longer received or when a link failure is reported by the link layer.

\section{A. Reactive protocols}

Upon link breakage detection, reactive protocols will basically issue a new route request to repair routes using that link. The route request is either initiated by the source of the route (in that case a notification of route error is sent to the source) or by the node detecting the link breakage (in that case, the term local route repair is often used). The policy used influences the $o_{r}$ parameter.

With $a N$ routes, there are $a N L$ active links. When an active link breaks, a route request has to be carried out for each destination reached through that link. This yields a total overhead of $\mu \times a N L \times N$ packets corresponding to a bandwidth utilization of $o_{r} \mu a L R Q_{r} N^{2}$.

This estimation may be pessimistic when several routes have identical destinations and the routes are repaired locally by the node detecting the link failure. Gains obtained from local repair may be integrated in the $o_{r}$ parameter.

\section{B. Proactive protocols}

It could be assumed that a proactive protocol would produce few additional control packets when a link breaks since the node detecting the breakage will probably be aware of another route to the destination. However in some situations, this alternative route may go through nodes that are not yet informed of the link breakage. This is a possible cause of routing loops. The easiest way to avoid such loops is to inform those nodes by first sending an additional topology broadcast packet. A very optimized protocol could unicast this topology packet to the destination. However, it would still be very difficult to technically ensure loop freedom. Moreover, longer routes might result until the next broadcast of a topology packet. A better optimization would consist in sending a topology broadcast packet with a reduced TTL (according to the distance from the destination in

\begin{tabular}{|c|c|c|}
\hline & \multicolumn{2}{|c|}{ Reactive protocols } \\
\hline & Packets & Bandwidth \\
\hline \multirow{4}{*}{$\begin{array}{l}\text { Fixed } \\
\text { Mobility }\end{array}$} & $\lambda o_{r} N^{2}+h_{r} N$ & $h_{r} H_{r} N+\lambda o_{r} R Q_{r} N^{2}$ \\
\hline & $o_{r} \mu a L N^{2}$ & $o_{r} \mu a L R Q_{r} N^{2}$ \\
\hline & Proac & ve protocols \\
\hline & Packets & Bandwidth \\
\hline $\begin{array}{l}\text { Fixed } \\
\text { Mobility }\end{array}$ & $\begin{array}{c}h_{p} N+o_{p} t_{p} N^{2} \\
o_{p} \mu A N_{p} N^{2}\end{array}$ & $\begin{array}{c}h_{p} H_{p} N+o_{p} t_{p} T_{p} N^{2} \\
o_{p} \mu A N_{p} T_{p} N^{2}\end{array}$ \\
\hline
\end{tabular}

TABLE VI

CONTROL TRAFFIC OVERHEAD IN AD-HOC NETWORK PROTOCOLS.

number of hops). For the purpose of this analysis, we will suppose that a node detecting a link breakage on a route will emit an additional topology broadcast packet.

Again, a given node is, on average, on $a L$ routes. As with reactive protocols, several routes may use the same outgoing link. However, the probability that the next hops for these routes are the same is certainly greater than the probability that the destinations for these routes are the same. We thus introduce the active next hop parameter $A N_{p}$ which is the average number of active next hops of a node. For a given protocol, this parameters depends on the nature of the network and the traffic. The total overhead will thus be $o_{p} \mu A N_{p} N^{2}$ packets corresponding to a bandwidth of $o_{p} \mu A N_{p} T_{p} N^{2}$.

Table VI summarizes the analysis of both protocol flavors control traffic overhead. They both include an $O\left(N^{2}\right)$ overhead. In the following section $\mathrm{V}$, we will compare our analysis to simulations from literature for AODV and DSR, as well as to simulations for OLSR, to validate our formulas.

\section{The ANALYTICAL MODEL AND SIMULATION RESUltS}

To our knowledge, the only published work related to our analysis and model are simulations of the various protocols [12], [7], [3], [6], [16].

The routing load defined in [6] and [16] does not allow an easy way to estimate the number of control packets. For that reason, we do not try to compare our analysis to these results.

This section will therefore compare and evaluate our proposed model with the simulations from [12], [7], [3] as well as to ns2 simulations of OLSR.

\section{A. Johansson et al. simulations}

[12] is close to the point of view of the present paper since a mobility metric is defined and simulations results are presented according to that metric. Like most simulations in literature, the "random waypoint" mobility model [3] is used. The metric for mobility is defined in terms of relative speed between nodes. [12] shows that the average number of link changes is approximately proportional to this mobility metric. It is thus consistent with our definition of mobility which is also proportional to the average number of link changes.

The simulations of AODV, DSR and DSDV in [12] show, that for AODV and DSR there is a close to linear relationship 
between control packet emissions and mobility. This confirms what is predicted by our $o_{r} \mu a L N^{2}$ factor as long as no congestion occurs. DSDV produces a constant number of control packets. This is due to the fact that DSDV does not broadcast additional topology packets in reaction to mobility. This fact can be expressed in our model by $A N_{p}=0$.

Unfortunately, only results for a fixed number of nodes (50) and fixed number of sources of traffic (15) are shown. Other scenarios are presented but again with fixed parameters preventing us from further comparing our model to the simulation results obtained in [12].

\section{B. Broch et al. simulations}

The most complete set of simulations of DSDV, TORA, DSR and AODV can be found in [3], which also is the paper originating the "random waypoint" mobility model. The traffic is produced by a fixed number of constant bit rate sources. Our parameter $a$ is thus simply the number of sources $(10,20$ or 30$)$ over the number of nodes (50).

As expected, the results for DSDV show, an approximately constant number of control packets. The results for TORA are quite unstable due to network congestion. As our model does not take congestion into account, we thus do not consider the TORA results.

Figure 1 extracts results presented in [3] for the highest mobility simulations (maximum speed of $20 \mathrm{~m} / \mathrm{s}$ ) obtained for AODV and DSR. The figure presented is modified in order to have the $x$-axis represent the average number of connectivity changes instead of the pause time originally used in [3]. This yields something proportional to our definition of mobility and allow to notice again the linearity of control overhead versus mobility.

Except the cases of very high mobility (about $>9000$ connectivity changes), the results from [3] confirm our model. The relatively low overhead cost for the highest mobility points could come from an implementation bounding the maximum rate of floodings per node or simply from congestion. In [3], this is not discussed further.

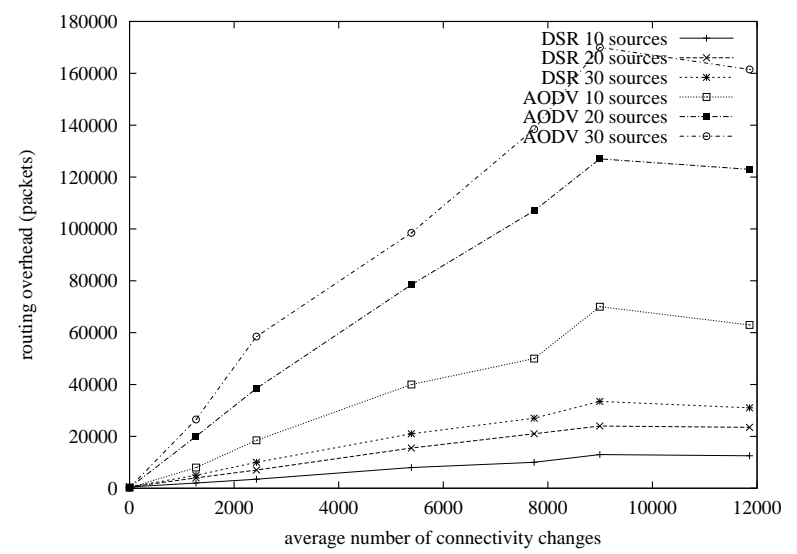

Fig. 1. Simulation results extracted from [3] with abscissae proportional to mobility (the number of connectivity changes can be expressed as $900 \times \mu \times$ $D N$ when the simulation lasts $900 s$ ).

Figure 2 shows the same results presented versus $\mu \times a$. For each simulation point $(S, C, n)$ where $n$ is the number of control packets observed, $C$ the number of connectivity changes and $S$ the number of sources, we have computed the point $(\mu \times a, n)$. The line that best approximates the points is also plotted.

This confirms the linearity of control traffic overhead with activity $a$ as predicted by our $o_{r} \mu a L N^{2}$ factor. The difference between AODV and DSR results may be explained by different $o_{r}$ factors: [3] points out that DSR makes extensive use of caching to limit the number of route requests and uses possibly a "non-propagating" route request for limiting the cost of a route request. We can infer from the curves that $o_{r}^{A O D V} \approx 2$ and $o_{r}^{D S R} \approx 0.4$ (the field geometry and the number of nodes implies $L \approx 3$ ).

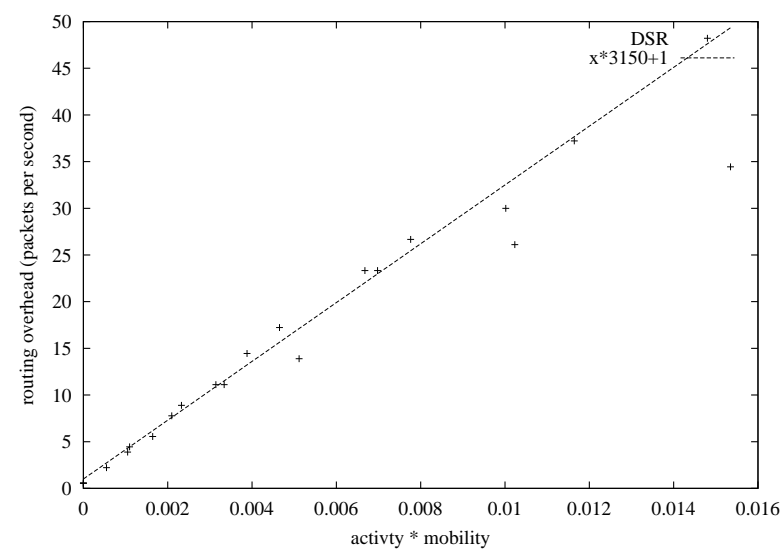

(a) DSR

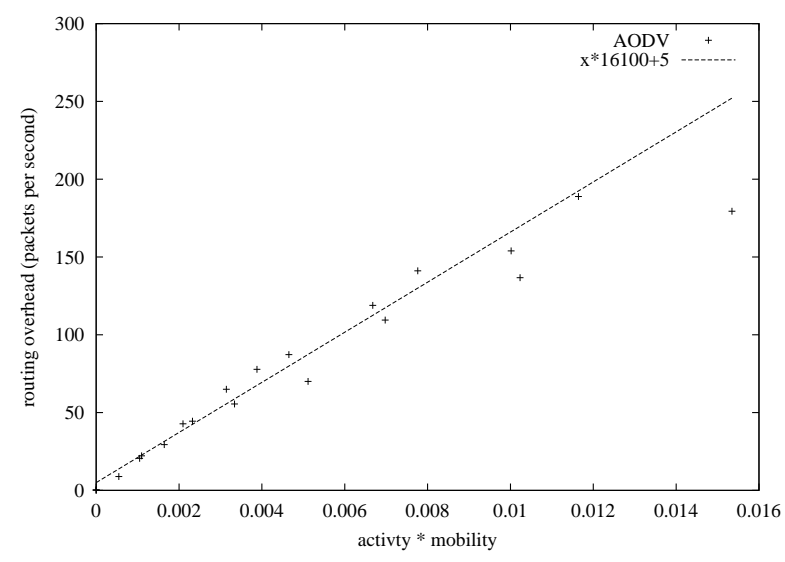

(b) AODV

Fig. 2. Simulation results taken from Figure 5 in [3] showing the number of control packets per second versus $\mu \times a$.

[7] points out additional caching results in lower packet delivery rate, but delivery rate is not predicted by our model.

\section{Das et al. simulations}

The simulations in [7] use the same simulation model as [3]. However in [7], a version of AODV employing an "expanding 
ring" technique to reduce the cost of route request floodings is used. Two sets of simulations are presented: one set with the same parameters as in [3] (the 50 nodes set), the other with 100 nodes and a larger field.

Comparing our analysis with the results found in [7], the simulations with 50 nodes agree again with our model for low data traffic rates but not for high data traffic rates where congestion occurs.

For the simulations in [7] with 100 nodes, our model and the simulation results are in complete agreement.

Figure 3 shows the simulation results for [7] with 100 nodes presented as number of control packets per second versus $\mu \times a$. (These curves are approximately deduced from the curves showing routing loads and packet delivery fractions in [7].) Thus in this case, we can infer from the figure $o_{r}^{D S R} \approx 2$ and $o_{r}^{A O D V} \approx 4$.

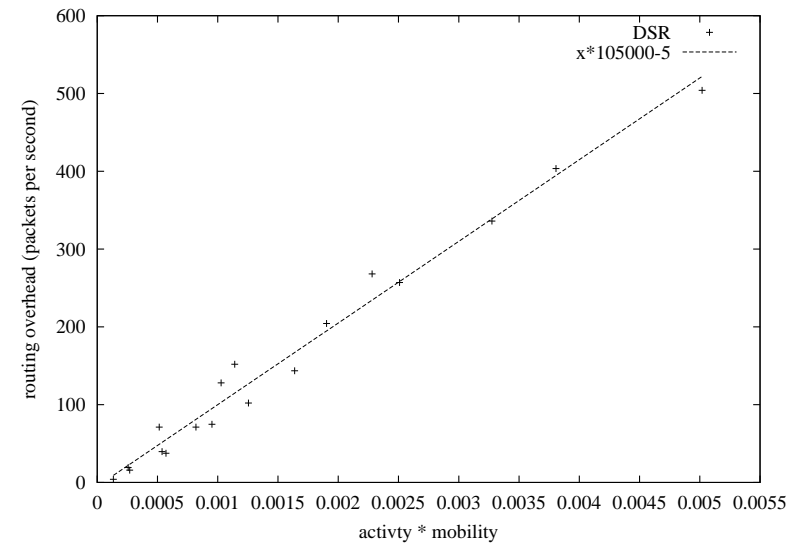

(a) DSR

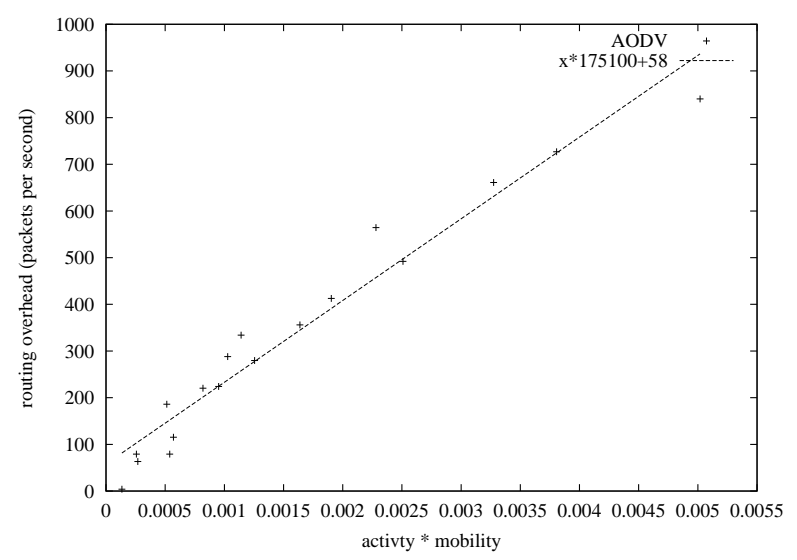

(b) AODV

Fig. 3. Simulation results extracted from Figure 4 and 5 in [6] showing the number of control packets per second versus $\mu \times a$.

\section{OLSR simulations}

In OLSR, all optimizations are made through the concept of multipoint relays (MPRs) [15], [11]. Each node selects a set of MPRs in its neighborhood and only those MPRs retransmit flooding packets transmitted by the node. Topology updates are made of MPR selector lists (each node sends the list of the nodes that have selected it as MPR). The active next hops must be multipoint relay selectors and their average number is thus equal to the average number of multipoint relays.

We have conducted ns 2 simulations of OLSR in the same framework as Johansson et al. simulations [3]. We first notice that control traffic generation does not depend on the data traffic characteristics. This is not surprising since reaction to mobility is made only with regards to MPR changes.

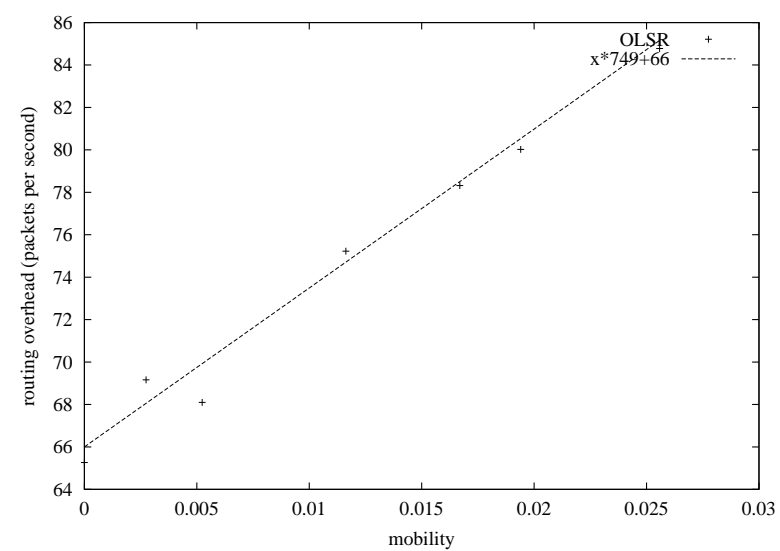

Fig. 4. Simulation results for OLSR showing the number of control packets per second versus $\mu$ (50 nodes moving in the $1500 \times 300$ field).

Figure 4 shows the simulation results. Since the data traffic rate is of no impact on the results, the $x$-axis represents only mobility. We again observe the linearity of control traffic overhead with respect to mobility. We can infer from the figure $o_{p}^{O L S R} A N_{p}^{O L S R} \approx 749 / 50^{2}=0.3$ and $o_{p}^{O L S R} \approx$ $66 / 0.2 / 50^{2}=0.13\left(t_{p}^{O L S R}=0.2\right)$.

To cross check these results, we have made simple simulations to infer the average number of MPRs of a node (which is equal to $A N_{p}^{O L S R}$ ) and the average number of emissions per MPR flooding (which is equal to $o_{p} * N$ ). In the $1500 \times 300$ field with $N=50$, we found $A N_{p}^{O L S R} \approx 2$ and $o_{p}^{O L S R} \approx$ $8.2 / 50=0.16$. For the $2200 \times 600$ field with $N=100$, we found $A N_{p}^{O L S R} \approx 3.2$ and $o_{p}^{O L S R} \approx 28 / 100$. (The average degree is around 10.5 for both scenarios. We also found $L \approx 3$ for the $1500 \times 300$ field with 50 nodes and $L \approx 4$ for the $2200 \times 600$ field with 100 nodes.)

There is a slight difference for the two estimations of $o_{p}^{O L S R}$ $(0.13$ and 0.16$)$ in the $1500 \times 300$ field with 50 nodes. This can be explained by the fact that only nodes having non-empty lists of MPR selector send topology updates. When there is no mobility (first estimation) some nodes do not send any topology update, yielding a better broadcast optimization factor. With mobility, nodes whose list becomes empty must still send an empty topology update to invalidate old information, yielding a slightly worse optimization factor. In the following, we will use the second estimation as it gives a sharp upper bound for the $1500 \times 300$ scenario $(820 \mu+82$ control packets per second instead of $749 \mu+66)$ and allows to give estimations for the $2200 \times 600$ scenario. 


\section{ANALYSIS OF THE MODEL'S PROTOCOL PARAMETERS}

Given the estimated parameters of the protocols, it is now possible to conduct comparisons for a wider range of scenarios. See the next Section where we compare DSR and OLSR for any activity and mobility pattern. This comparison is based on the estimation of the protocol parameters through simulation of a few activity and mobility patterns. Indeed, our approach would benefit from inferring the protocol parameters of the model through analysis. This would allow to compare protocols in an even larger set of scenarios.

However, inferring some protocols parameters of the model from analysis (namely $o_{r}, o_{p}$ and $A N_{p}$ ) is rather intricate. For example, a very simple reactive protocol systematically flooding a route request from the source when a route fails and always expected the route reply from the destination would have $o_{r}=1+L / N$ as mentioned before. However the analysis of the number of route reply packets becomes intricate when intermediate nodes may also reply to the route request. It can become even more complex when techniques such as expanded ring or local route repair are used. Such analysis is reserved for future work.

Concerning proactive protocols, the main issue is to analyze the size of the part of the topology that is broadcasted. In the OLSR case, $o_{p}$ and $A N_{p}$ can be roughly inferred from the average number of multipoint relays $R$ per node. A node retransmits a broadcast packet if it is multipoint relay from the last emitter of the received packet which occurs with rough probability of $R / \Delta . A N_{p}$ is simply equal to $R$. The interested reader may find such analysis of OLSR in [10].

\section{CONTROL TRAFFIC OVERHEAD COMPARISON}

We observe that among the reactive protocols, AODV generates more control traffic than DSR. We have thus chosen to compare the proactive protocol OLSR with DSR.

\section{A. Tolerance to mobility}

Let $R T T$ be the time needed for a round trip packet to go from a source to a destination and back to the source. To be able to route packets from the source to the destination, a reactive protocol needs the route to remain alive at least $R T T$ long. (Indeed, RTT is a lower bound on the time needed to complete a route request.) When the length of a route is $L$, some link on the route breaks at the rate of $L \mu$. The maximal allowable mobility is thus $\mu_{r}=1 / L R T T$ for reactive protocols.

With proactive protocols, when a link breaks, a topology broadcast packet is sent. Packets from the source with valid route will thus come back after RTT time. The maximum allowed mobility is thus $\mu_{p}=1 / R T T=L \mu_{r}$. This shows that proactive protocols have better tolerance to mobility as long as the limiting factor is the round trip time and not the bandwidth.

To compare the protocols, we should thus consider $0 \leq \mu \leq$ $1 / L R T T$. Notice that $\mu=1 / L R T T$ represents a very high mobility since for $R T T=100$ millisecond and $L=10$, this gives $\mu=1$, meaning that the average duration of a link is then 1 second. In the mobility model of [3], this corresponds to an average node speed in the magnitude of 100 meters/sec.

\section{B. Parameter values for comparisons}

To compare two protocols, we need to determine some parameters. First of all, some protocol parameters are known: $h_{p}^{O L S R}=0.5$ and $t_{p}^{O L S R}=0.25$ and $h_{r}^{D S R}=0$. Packet size parameters are analyzed bellow. Other protocol parameters are deduced from the simulations presented in section $\mathrm{V}$.

We will compare the two protocols in terms of $\mu$ and $a$.

The final parameter is $\lambda$, which models traffic creation. Considering typical traffic such as web browsing, it is natural to suppose that a given source of traffic changes its destination from time to time - say every 60 seconds. Thus for this comparison, we use $\lambda=a / 60$ (notice that the overhead thus introduced for DSR is neglectable as soon as $\mu \geq 0.05$ ).

To compare bandwidth utilization, we have to estimate the overhead of packet emission. We will suppose the use of IEEE 802.11 MAC layer [18]. The average duration of the backoff is 310 microseconds, the DIFS interval is 50 microseconds and the synchronization overhead is at least 96 microseconds. Supposing $1 \mathrm{Mb} / \mathrm{sec}$ data rate, this corresponds to an overhead of 456 bits (or equivalently 57 bytes) per packet emission. Moreover each packet must contain a MAC header of 34 bytes and an IP header of 20 bytes.

A DSR route request will include 8 additional bytes plus at list two addresses. An OLSR topology update will include 20 additional bytes plus $A N_{p}$ addresses. An OLSR hello message will include 36 additional bytes plus $\Delta$ addresses. Considering IPv4 addressing, we will thus suppose: $R Q_{r}^{D S R}=127$, $T_{p}^{O L S R}=131+4 A N_{p}$ and $H_{p}=147+4 \Delta$.

\section{Comparison equation}

Our aim in this section is to identify the set of scenario parameters where the reactive protocol performs either significantly better or significantly worse than its proactive counterpart. Restricting our analysis to control traffic overhead and taking into account the results of our previous analysis, this consists into identifying the areas where:

$127\left(\mu L+\frac{1}{60}\right) o_{r}^{D S R} a \lesseqgtr\left((73.5 / N+2 d)+o_{p}^{O L S R}(.2+\right.$ $\left.\left.\mu A N_{p}^{O L S R}\right)\left(131+4 A N_{p}^{O L S R}\right)\right)$

\section{Planar free space model}

In this model, as in the simulations presented before, the nodes are randomly placed on the plane. Two nodes may communicate if their distance is less than the radio range. This model is also known as the random unit graph model.

Using our analytical model, we can now compare DSR and OLSR for any mobility and data traffic patterns. Figure 5 and figure 6 presents the regions favorable to each protocol in the plane $\mu \mathrm{x} a$.

We find that high data traffic favors OLSR, and we also notice that the area where OLSR is of preference is larger in the big network. On the other hand, for low traffic rates, DSR yields the better results.

We notice, that the usually admitted paradigm stating that reactive protocols behave better with regard to control traffic overhead when mobility increases proves to be wrong. While it is correct for low traffic (in number of routes), when the traffic in the network grows beyond a certain limit, the proactive 


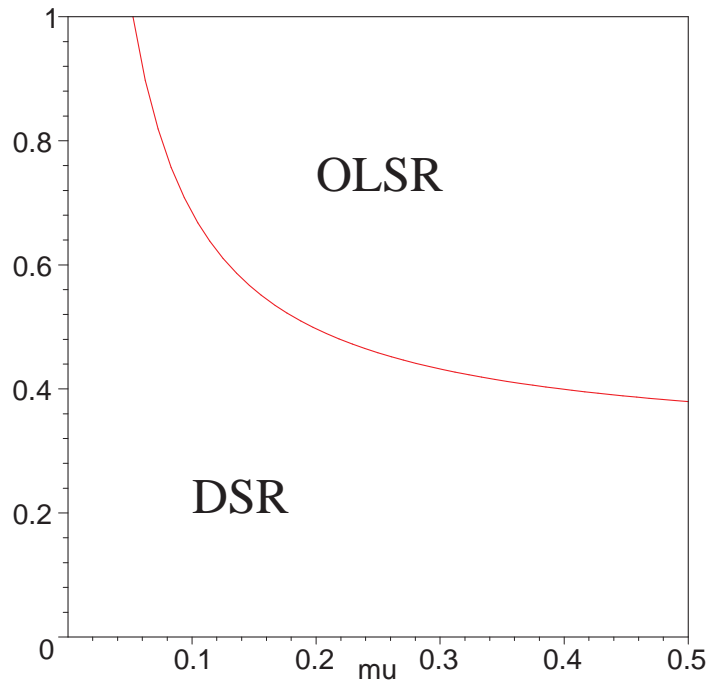

Fig. 5. Comparison of OLSR and DSR control overheads in the free space model. The abscissae is $\mu$ and the ordinate is $a . N=50,1500 \times 300$ field.

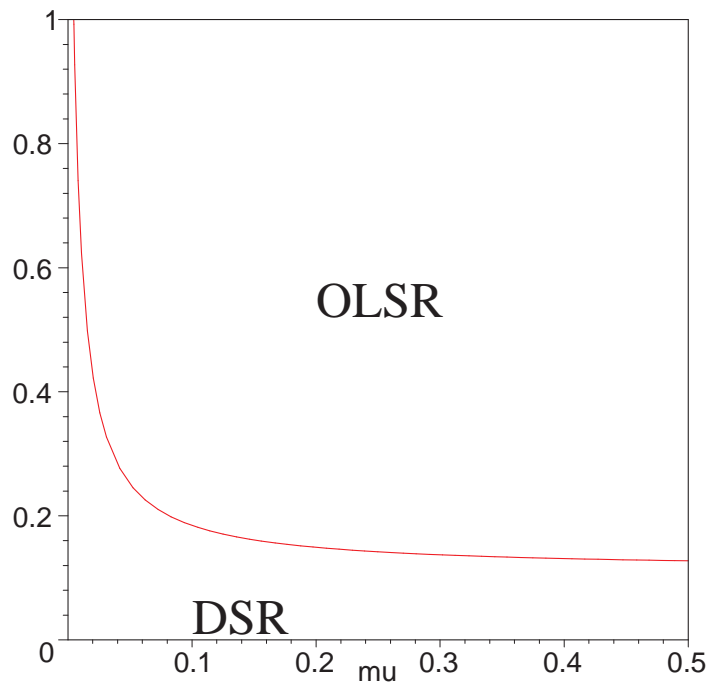

Fig. 6. Comparison of OLSR and DSR control overheads in the free space model. The abscissae is $\mu$ and the ordinate is $a . N=100,2260 \times 600$ field.

protocols are of preference. We call this limit the activity limit $a_{l}$.

\section{E. Rough high mobility asymptotic}

We can roughly estimate the activity limit between proactive and reactive protocols for high mobility. Comparing a proactive protocol with a reactive protocol, we can first suppose that the size of control packets is similar for both (this assumption is reasonable when the overhead of sending a packet is quite high as with IP over IEEE 802.11). For high mobility the comparison equation thus becomes: $\mu L o_{r} a \lesseqgtr \mu A N_{p} o_{p}$. For the same broadcast optimization we obtain an asymptotic activity limit $a_{l}=A N_{p} / L$. Notice that a reactive protocol can achieve exactly the same broadcast optimization factor as a proactive protocol (e.g. DSR can employ hello messages and perform flooding using the MPR optimization of OLSR) thus allowing to suppose $o_{r}=o_{p}$. We note that reactive protocols are better suited as long as $a N L \leq A N_{p} N$. Notice that $a N$ is the number of routes and $A N_{p} N$ is the number of active links. This means that reactive protocols are better suited (with high mobility) as long as routes do not share links.

\section{CONCLUSION}

We have proposed an analytical model that allows us to describe and reason on MANET routing protocols. The model is parameterized such that it can accommodate any proactive or reactive routing protocol. We have found, when applying the model to scenarios where simulation studies exist, that the model accurately reflects the simulations.

We notice that the difficult task is to identify the correct values for the protocol parameters. However once these are estimated, it is possible to compare different protocols in a very wide range of mobility versus traffic patterns.

To extend our analysis, one should also consider overhead due to non-optimal routes.

Our model allows us to detect a fundamental limit between proactive and reactive approaches for high mobility tolerance. This limit shows, that proactive protocols are better suited as soon as a significant number of links can be reused for several routes.

\section{REFERENCES}

[1] G. Aggelou and R. Tafazolli. Rdmar: A bandwidth-efficient routing protocol for mobile ad hoc networks. In Proceedings of ACM MobiCom99/WoWMoM99, August 1999. Washington, USA.

[2] B. Bellur and RG. Ogier. A reliable, efficient topology broadcast protocol for dynamic networks. In INFOCOM'99, March 1999.

[3] J. Broch, D.A. Maltz, D.B. Johnson, Y.-C. Hu, and J. Jetcheva. A performance comparison of multi-hop wireless ad hoc network routing protocols. In MobiCom'98, October 1998. Dallas.

[4] T. Clausen, P. Jacquet, A. Laouiti, P. Muhlethaler, A. Qayyum, and L. Viennot. Optimized link state routing protocol. In IEEE INMIC Pakistan, 2001.

[5] M. S. Corson and J. Macker. Mobile ad hoc networking (MANET): Routing protocol performance issues and evaluation considerations. RFC 2501, January 1999.

[6] S. Das, R. Castaneda, and J. Yan. Simulation based performance evaluation of mobile. ACM/Baltzer Mobile Networks and Applications (MONET), pages 179-189, July 2000.

[7] Samir R. Das, Charles E. Perkins, and Elizabeth M. Royer. Performance comparison of two on-demand routing protocols for ad hoc networks. In Proceedings of the IEEE Conference on Computer Communications (INFOCOM), March 2000. Tel Aviv, Israel.

[8] S. Datta, I. Stojmenovic, and J. Wu. Internal node and shortcut based routing with guaranteed deliv ery in wireless networks. In Proc. IEEE Int. Conf. on Distributed Computing and Systems, April 16-19 2001. Phoenix, AR.

[9] Z.J. Haas and M.R. Pearlman. Providing ad-hoc connectivity with the reconfigurable wireless networks. In Charles Perkins, editor, Ad Hoc Networks. Addison Wesley Longman, 2000.

[10] P. Jacquet, A. Laouiti, P. Minet, and L. Viennot. Performance analysis of olsr multipoint relay flooding in two ad hoc wireless network models. In The second IFIP-TC6 NETWORKING Conference, may 2002. Pise.

[11] P. Jacquet, A. Laouiti, P. Minet, and L. Viennot. Performance of multipoint relaying in ad hoc mobile routing protocols. In IFIP Networking 2002, may 2002. Pisa.

[12] P. Johansson, T. Larsson, N. Hedman, B. Mielczarek, and M. Degermark. Scenario-based performance analysis of routing protocols for mobile ad hoc networks. In MobiCom'99, August 1999. Seattle.

[13] David B. Johnson and David A. Maltz. Dynamic source routing in ad hoc wireless networks. Mobile Computing, 5:153-181, 1996. Kluwer Academic Publishers.

[14] Scott Corson Joseph Macker. Mobile adhoc networking and the ietf. ACM Mobile Computing and Communications Review, 1998. 
[15] A. Laouiti, A. Qayyum, and L. Viennot. Multipoint relaying: An efficient technique for flooding in mobile wireless networks. In 35th Annual Hawaii International Conference on System Sciences (HICSS'2001). IEEE Computer Society, 2001.

[16] S.-J. Lee. Routing and Multicasting Strategies in Wireless Mobile Ad hoc Networks. PhD thesis, University of California, 2000.

[17] S.-J. Lee, M. Gerla, and C.-C. Chiang. On-demand multicast routing protocol. In Proceedings of IEEE WCNC'99, pages 1298-1302, September 1999. New Orleans, LA.

[18] LAN MAN Standards Committee of the IEEE Computer Society. Wireless lan medium access control (mac) and physical layer (phy) specifications, June 1997. IEEE 802.11.

[19] V. Park and M. S. Corson. A highly adaptive distributed routing algorithm for mobile wireless networks. In Proc. IEEE INFOCOM '97. Kobe, Japan, 1997.

[20] Charles E. Perkins and Elizabeth M. Royer. Ad-hoc on-demand distance vector routing. In Proceedings of the 2nd IEEE Workshop on Mobile Computing Systems and Applications, pages 90-100, February 1999. New Orleans, LA.

\section{ABOUT THE AUTHORS}

\section{Thomas Heide Clausen}

Received his engineering degree (cand.polyt, M.Sc.) from the Department of Computer Science, Aalborg University, where he is currently a PhD-student. He's currently visiting the Hipercom team at INRIA where he works on wireless networks.

Philippe Jacquet

Research Director and leader of the Hipercom team in INRIA, Docteur Habilit, graduated from Corps des Mines in 1984. Main topics: information theory, mobile ad hoc networks.

\section{Laurent Viennot}

Laurent Viennot has graduated from Ecole Polytechnique in Palaiseau. He has then completed a doctorate in Computer Science at University Paris 7 about graph algorithmics. He then joined the Hipercom team at INRIA to work on wireless networks. 